\title{
The Effect of Prandial Insulin Applied for Fat Protein Units on Postprandial Glucose Excursions in Type 1 Diabetes Patients with Insulin Pump Therapy: Results of a Randomized, Controlled, Cross-Over Study
}

\author{
Authors

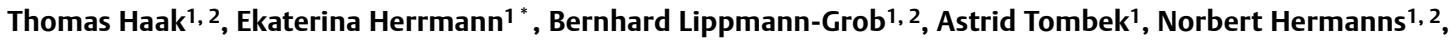 \\ Michael Krichbaum²
}

Affiliations

1 Diabetes Clinic, Bad Mergentheim, Gemany

2 FIDAM - Research Institute Diabetes Academy, Mergentheim, Gemany

Key words type 1 diabetes, insulin pump therapy, insulin calculation, fat protein units

received 16.09 .2020

revised 13.03.2021

accepted 17.03.2021

published online $\quad 20.04 .2021$

Bibliography

Exp Clin Endocrinol Diabetes 2022; 130: 262-267

DOI 10.1055/a-1474-8193

ISSN 0947-7349

(c) 2021. Thieme. All rights reserved.

Georg Thieme Verlag KG, Rüdigerstraße 14,

70469 Stuttgart, Germany

Correspondence

Prof. Dr. med. Thomas Haak

Diabetes Center Mergentheim

Theodor-Klotzbücher-Str. 12

D-97980

Bad Mergentheim

Germany

Tel.: + 497931 549-101

haak@diabetes-zentrum.de

\begin{abstract}
Objective This randomized cross-over study aimed to compare different algorithms for calculating prandial insulin considering the fat and protein content of a standardized meal in type 1 diabetes patients using insulin pump therapy (CSII). Methods Twenty-six patients received a standardized evening meal for three consecutive days using different algorithms for insulin dose adjustment: A) exclusive consideration of carbohydrate content without considering fat-protein content, B) high-dose algorithm considering additional insulin for fat protein units (FPUs) with the same factor as for carbohydrates, and C) low-dose algorithm considering additional insulin for FPUs with half the factor as for carbohydrates. The primary outcome was the proportion of interstitial glucose values in the target range ( $\geq 70$ to $\leq 180 \mathrm{mg} / \mathrm{dl}$ ) during the post-prandial 12 -hour follow-up period. Secondary outcomes were the occurrence of hypo- and hyperglycemic episodes and the coverage with carbohydrates for treatment of hypoglycemia.

Results The percentage of glucose values in the target range was significantly higher when fat-protein content was not considered, whereas, in the hyperglycemic range, it did not differ significantly among the three groups. The percentage of hypoglycemic glucose values were the highest in the groups considering fat-protein content and lowest in the group not considering FPUs with no significant difference between the two groups in terms of FPUs.

Conclusions In adult type 1 diabetes patients using CSII, it is not recommended to consider a high fat and protein content in the diet when calculating prandial insulin dosage with the selected algorithms, as this increases the risk of hypoglycemia disproportionately.
\end{abstract}

\section{Introduction}

Usually, carbohydrates consumed in the diet are regarded as the decisive factor for an increase in postprandial glucose excursion. Consequently, with intensified insulin therapy, the amount of carbohydrates is used to calculate the prandial insulin dosage by mul-

E. Herrmann actually is affiliated with Medicover Oldenburg MVZ. tiplying the amount of carbohydrate content with an individual carbohydrate-insulin ratio (CIR) [1,2]. However, besides the amount of carbohydrates, the fat and protein contents of a meal have also been demonstrated to influence the postprandial glucose excursion [3-6]. A high fat and protein content often delays gastric emptying and the absorption of carbohydrates from the intestine into the blood, resulting in a delayed increase in post-prandial glucose. High protein and fat content in meals challenge the match 
of prandial insulin action and post-prandial glucose. This delayed absorption of carbohydrates can be countered therapeutically by splitting the insulin bolus or delaying the infusion of prandial insulin as offered in modern insulin pump therapy $[1,2,7]$.

Bell et al. found an impact of a high-fat content of a meal on postprandial glucose excursions in six out of seven reviewed studies, mainly indicating higher post-prandial glucose excursions after fatty meals [3]. It is speculated that beyond the delayed glucose absorption dietary fat might induce higher levels of free fatty acids and this, in turn, could cause insulin resistance, requiring higher insulin doses for optimizing postprandial glucose control [8].

Protein-rich meals can also have a hyperglycemic effect on postprandial glucose control. In particular, high amounts of proteins $(>75 \mathrm{~g}$ ) or the addition of a moderate amount of fat and protein have been associated with higher postprandial glucose levels $[9,10]$.

These data led to the assumption that high fat and protein content in a meal requires additional prandial insulin. To standardize insulin dosing for fat and protein, "fat protein units" (FPU) were introduced by Ewa Pańkowska in 2009 [11]. One FPU corresponds to the amount of fat and/or protein containing $100 \mathrm{kcal}$ energy.

For fat- and/or protein-rich meals, whether and which amount of additional prandial insulin should be administered remains debatable [3,4]. Pańkowska et al. [12,13] recommended using the same amount of insulin for an FPU as for one carbohydrate unit, an algorithm that has been used in many other studies $[3,4,14]$, while another algorithm suggests using only half the amount of insulin for an FPU as is used for one carbohydrate unit [15]. However, Pańkowska et al. studied only children with type 1 diabetes [12, 13], and Lee et al. [15] investigated different means of bolus administration (normal bolus vs. dual wave bolus) without randomization.

Many studies about insulin adjustment after the intake of fatand/or protein-rich meals have been performed in children, who have a rather short diabetes duration and rather low insulin demand. Therefore, we conducted a randomized controlled study and investigated the two above-mentioned algorithms in comparison to no FPU insulin adjustment in an adult sample of type 1 diabetes patients. We expected that the adjustment of prandial insulin doses for FPU would optimize post-prandial glycemic control compared to that in a non-adjusted condition. We selected the percentage of post-prandial glucose values in the range of $\geq 70 \mathrm{mg} / \mathrm{dl}$ and $\leq 180$ $\mathrm{mg} / \mathrm{dl}$ [16] as the primary outcome variable to "punish" hyper- and hypoglycemic glucose excursions equally.

\section{Material and Methods}

\section{Study design and participant recruitment}

This study was a monocenter, open-label, parallel, randomized, controlled crossover trial. The study design is presented in $\mathbf{~ F i g - ~}$ ure 1.

The study was carried out in an inpatient setting at the Diabetes Center Mergentheim. Study participants were eligible if they had type 1 diabetes mellitus. Additional inclusion criteria were treatment with insulin pump therapy (CSII), age $\geq 18$ to $\leq 70$ years, and sufficient knowledge of the German language. Exclusion criteria were severe general illness, severe psychiatric illness, gastroenterological disease, impaired kidney function, and heart attack, stroke/TIA, or vascular surgery within the past six months. Participants with changes in basal rate during the experimental days were also excluded. Before inclusion, both oral and written information about the study was provided to all participants, who then provided written informed consent.

The study was reviewed and approved by the federal agency (Federal Institute for Pharmaceuticals and Medical Products, BfArM), and the local ethics committee (State Medical Association of Baden-Württemberg) and is registered in the EU Clinical Trials Register (EudraCT Number 2017-001807-62).

\section{Procedures}

On three consecutive days, the study participants received a standardized carbohydrate-reduced, high-fat, high-protein evening meal, the Viennese-style chicken schnitzel breaded with potato gratin and salad (702.6 kcal, $52.7 \mathrm{~g}$ fat, $33.8 \mathrm{~g}$ protein, $23.3 \mathrm{~g}$ carbohydrates). The test meal contained 2.3 carbohydrate units (CU) and 6 fat protein units (FPUs). The prandial insulin dose was calculated using three different algorithms as defined in the clinical investigation plan. The sequence of the three conditions was randomized.

\section{Algorithms for Insulin Dose Adjustment}

\section{Condition A (non-FPU)}

Standard adjustment considering only the carbohydrate content of the meal with an insulin dosage based on the carbohydrate-toinsulin ratio (CIR). Insulin was delivered as a dual bolus (50\% before eating, $50 \%$ delayed over four hours).

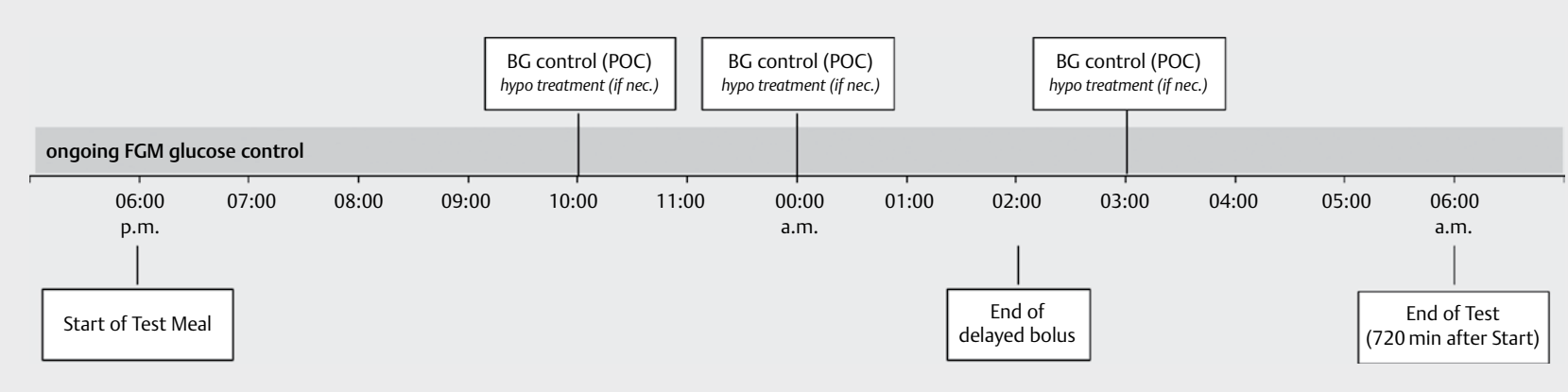

Fig. 1 The study flow chart 


\section{Condition B (FPU 100\%)}

This algorithm took into account carbohydrates as well as fat and protein content. The carbohydrate content was covered by the individual CIR. For each FPU, a factor of $100 \%$ of the CIR was used. To cover carbohydrates, insulin was delivered as a dual bolus ( $50 \%$ before eating; $50 \%$ delayed over eight hours), and to cover FPU, insulin was delivered as a delayed bolus over eight hours.

\section{Condition C (FPU $50 \%$ )}

This algorithm considered carbohydrates as well as fat and protein content. The carbohydrate content was covered by the individual CIR. For each FPU a factor of $50 \%$ of the CIR was used. To cover carbohydrates, insulin was delivered as a dual bolus ( $50 \%$ before eating; $50 \%$ delayed over eight hours), and to cover FPU, insulin was delivered as a delayed bolus over eight hours.

\section{Blood Glucose Control}

Before the start of the test meal (at 6:00 p.m.), blood glucose was determined based on a routine capillary blood glucose measurement with a point-of-care (POC) blood glucose meter (Glucometer PRO, BST Biosensor Technology GmbH, Berlin, Germany). The prandial insulin doses were adjusted based on this POC measurement. The course of glucose after the start of the test meals was determined using a continuous interstitial glucose measurement system (FreeStyle Libre, Flash Glucose Monitoring System (FGM), Abbott, Wiesbaden, Germany). Interstitial glucose values were scanned at least before the start of the test meals, before going to bed, in the morning after the test meals, and when routine blood glucose measurements were done. For safety reasons four additional routine blood glucose measurements provided for inpatient treatment were performed at 10:00 p.m., 00:00 a.m., 3:00 a.m., and 6:00 a.m. using a POC blood glucose meter. Glucose values that were too high (up to $150 \mathrm{mg} / \mathrm{dl}$ above the starting value before the test meal) were not corrected unless ketone bodies were additionally detected in the urine (acetone test two-fold or three-fold positive). If blood glucose was too low at the nighttime blood glucose checks, the participants received $10-20 \mathrm{~g}$ of fast absorbable rescue carbohydrates.

\section{Follow-up}

In the morning of the day following the test meal, the glucose data of the FGM system and the insulin data of the CSII systems were readout. The occurrence of $\mathrm{POC}$ confirmed level 1 hypoglycemia ( $<70 \mathrm{mg} / \mathrm{dl}$ ) or hyperglycemia (> $180 \mathrm{mg} / \mathrm{dl}$ ), and the amount of rescue carbohydrates was recorded. The occurrence of (severe) adverse events (AEs/SAEs) or (severe) adverse device effects (ADEs/ SADEs) were also recorded.

\section{Outcomes}

The primary study outcome was the percentage of interstitial glucose values in the target range ( $\geq 70$ to $\leq 180 \mathrm{mg} / \mathrm{dl}$ ) within a period of 12 hours after taking the test meal.

Secondary study outcomes were the percentage of interstitial hypoglycemic glucose values ( $<70 \mathrm{mg} / \mathrm{dl}$ ) and hyperglycemic values (> $180 \mathrm{mg} / \mathrm{dl}$ ) within 12 hours after the test meal. The percent- age of POC confirmed level 1 hypoglycemia ( $<70 \mathrm{mg} / \mathrm{dl}$ ) or hyperglycemia ( $>180 \mathrm{mg} / \mathrm{dl}$ ) and the amount of rescue carbohydrates for treating POC-confirmed hypoglycemia.

\section{Statistical Methods}

\section{Statistical analysis}

In this study, a total of 26 people with type 1 diabetes were examined. The power consideration of such a sample size statistically confirmed an effective size of 0.7 standard deviations with a power of $\beta=0.8$ (two-sided $\alpha=0.025$; due to alpha adjustment for multiple testing).

The full analysis dataset consisted of participants who took part in all three test conditions. The primary and secondary outcomes were analyzed by repeated variance analysis controlled for order effects. Specific contrasts between single test conditions were determined if the overall test was significant. The significance level for all tests was $p<0.05$.

\section{Randomization and masking}

Randomization was done centrally at the study coordinating center by staff who were not involved in the recruitment or treatment of study participants. A randomization sequence was generated with SYSTAT 12.0 with a $1: 1: 1$ allocation.

The study physician received sealed envelopes with the respective allocation of the order of prandial insulin doses. After obtaining written informed consent, the envelope was opened. Masking of study participants and study personnel was not feasible because of the nature of the intervention.

\section{Results}

\section{Study participants}

The study was carried out at the Diabetes Center Mergentheim from September 2017 to January 2018. A total of 35 participants who met all inclusion criteria and who had given informed consent were recruited. One participant was excluded again due to an unstable basal rate (exclusion criterion). Four participants withdrew their consent to continue the study before they went through all three test conditions. Thirty participants completed the study as per the study requirement (participation in all three test conditions). Four of these participants had to be excluded from analysis because of protocol violations regarding insulin administration or because of the lack of sensor data (flash glucose monitoring, FGM) during the outcome phase (device failure). - Figure $\mathbf{2}$ shows the flow diagram of patient recruitment and data analysis.

- Table 1 shows the demographic and diabetes-specific characteristics of the sample. Study participants were middle-aged adults with long-standing type 1 diabetes and under insulin pump therapy for several years. With a mean HbA1c of $8.3 \%$, glycemic control needed improvement. $>$ Table 2 summarizes the primary and secondary outcome glucose parameters during the 12-hour follow-up period. - Figure $\mathbf{3}$ shows the glucose course under the three test conditions between 5:00 p.m. (pre-prandial phase) and 6:00 a.m. the next day. 


\section{Glycemic outcomes}

The bolus administered according to the above-described algorithms was $3.45 \pm 1.38 \mathrm{IU}$ for algorithm $A$ (non-FPU), $10.90 \pm 4.60$ IU for algorithm B (FPU $100 \%$ ), and $7.0 \pm 2.67 \mathrm{IU}$ for algorithm C (FPU $50 \%$ ). Mean pre-prandial glucose values at the beginning of the test meals were comparable among groups $(p=0.593)$.

There was a significant difference between the three test conditions considering the primary outcome percentage of time in the range of $\geq 70 \mathrm{mg} / \mathrm{dl}$ to $\leq 180 \mathrm{mg} / \mathrm{dl}$. However, the direction of the difference was in contrast to the original expectation. The adjust-

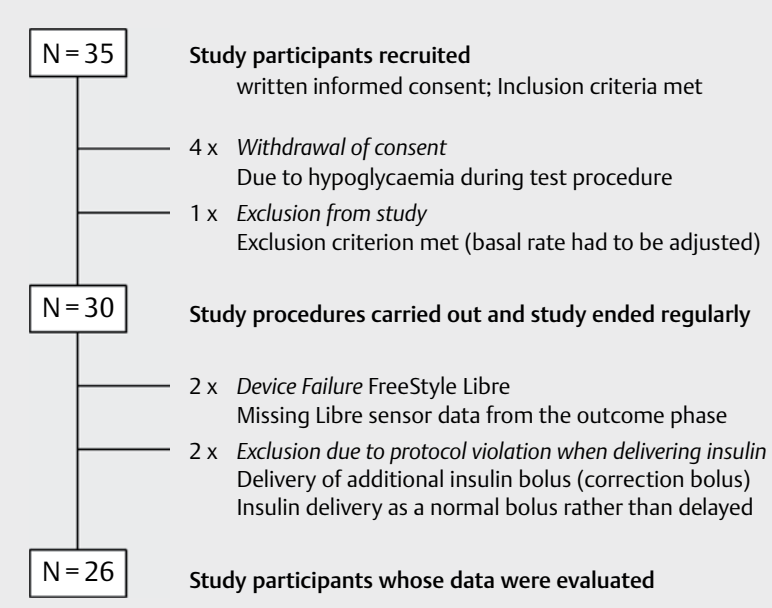

- Fig. 2 Glucose course under the three test conditions between 5:00 p.m. (pre-prandial phase) and 6:00 a.m. the next day (confidence intervals that do not intersect mean a significant difference between the corresponding conditions).

- Table 1 Demographic and diabetes-specific characteristics of the study sample $(\mathrm{N}=26)$.

\begin{tabular}{|l|l|l|}
\hline Characteristic & Mean \pm SD resp. N (\%) & Range \\
\hline Age (years) & $40.8 \pm 14.0$ & $18.2-63.3$ \\
\hline Female gender $(\mathrm{N} \%)$ & $13(50)$ & \\
\hline $\mathrm{BMI}\left(\mathrm{kg} / \mathrm{m}^{2}\right)$ & $27.2 \pm 5.0$ & $19.4-42.3$ \\
\hline $\mathrm{HbA} 1 \mathrm{c}(\%)$ & $8.3 \pm 1.5$ & $5.7-11.6$ \\
\hline Diabetes duration (years) & $22.8 \pm 12.0$ & $7.8-47.8$ \\
\hline CSIl therapy duration (years) & $7.6 \pm 7.7$ & $0.0-29.8$ \\
\hline
\end{tabular}

ment of insulin with $100 \%$ CIR per FPU showed a significantly less time in the range than with non-adjustment of prandial insulin for FPU. The adjustment with 50 \% CIR per FPU also showed a lower percentage of glucose values in the range of $70 \mathrm{mg} / \mathrm{dl}$ to $180 \mathrm{mg} /$ $\mathrm{dl}$ than with non-adjustment of prandial insulin for FPU, but this difference was not significant. The proportion of hypoglycemic glucose values was significantly higher if prandial insulin was adjusted for FPU with $100 \%$ CIR and $50 \%$ CIR per FPU (30.8\% and $21.8 \%$ vs. $5.5 \%$ hypoglycemic values). The nadir of glucose was reached at 3:00 a.m. and 4:00 a.m. in conditions B and C, respectively.

The proportion of hyperglycemic values was not significantly affected when considering the FPU for prandial insulin adjustment. The high prevalence of biochemical hypoglycemia captured with ongoing FGM in conditions B and C compared to condition A was corroborated by a significantly higher number of nocturnal confirmatory POC blood glucose measurements $<70 \mathrm{mg} / \mathrm{dl}$. At the same time, significantly more hypoglycemia rescue carbohydrates were administered to stabilize the glucose level in conditions B and $C$ than in condition $A$. The only outcome which was in favor of the adjustment of prandial insulin for FPU was the mean nocturnal postprandial glucose level, which was significantly lower in conditions $\mathrm{B}(107.9 \mathrm{mg} / \mathrm{dl})$ and C (116.4 mg/dl) compared to non-adjustment for FPU (144.5 mg/dl).

\section{Safety}

No serious adverse event occurred during the study. The nocturnal monitoring of blood glucose values ensured that no severe hypoglycemia event demanding the injection of glucose or glucagon occurred.

\section{Discussion}

This study clearly demonstrates that additional prandial insulin for a high-fat and -protein content of a meal does not have an advantage for a higher postprandial time in range. In contrast, additional insulin for FPUs resulted in a higher risk of postprandial hypoglycemia. The same was true for a reduced dose of additional prandial insulin for FPU in terms of the time in range and hyperglycemia. Although lower mean nocturnal glucose levels were within the range of $70-180 \mathrm{mg} / \mathrm{dl}$ when insulin was given for FPUs, one must note that these were obtained by a very high proportion of hypoglycemic values. The results from an FGM device were confirmed

- Table 2 Primary and secondary outcome glucose parameters during the 12-hour follow-up period ( $N=26)$.

\begin{tabular}{|c|c|c|c|c|c|c|}
\hline Glucose parameter & $\begin{array}{l}\text { Condition A } \\
\text { (non FPU) }\end{array}$ & $\begin{array}{l}\text { Condition B } \\
\text { (FPU 100\%) }\end{array}$ & $\begin{array}{l}\text { Condition C } \\
\text { (FPU } 50 \% \text { ) }\end{array}$ & $\begin{array}{l}\text { overall } \\
\text { p }\end{array}$ & $\begin{array}{l}\text { p Difference } \\
\text { Conditions } \\
\text { A/B }\end{array}$ & $\begin{array}{l}\text { p Difference } \\
\text { Conditions } \\
\text { A/C }\end{array}$ \\
\hline \multicolumn{7}{|l|}{ Primary Outcome } \\
\hline Percentage of glucose values in range $>70$ to $\leq 180 \mathrm{mg} / \mathrm{dl}$ & $73.84 \pm 23.46$ & $56.40 \pm 15.89$ & $64.84 \pm 24.01$ & 0.043 & 0.241 & 0.930 \\
\hline \multicolumn{7}{|l|}{ Secondary Outcomes } \\
\hline Percentage of glucose values $\leq 70 \mathrm{mg} / \mathrm{dl}$ & $5.54 \pm 11.30$ & $30.80 \pm 13.99$ & $21.81 \pm 20.16$ & $<0.001$ & $<0.001$ & 0.002 \\
\hline Percentage of glucose values $>180 \mathrm{mg} / \mathrm{dl}$ & $20.62 \pm 24.91$ & $12.80 \pm 14.14$ & $13.35 \pm 20.80$ & 0.260 & 0.441 & 0.531 \\
\hline Mean post-prandial glucose values in $\mathrm{mg} / \mathrm{dl}$ & $144.47 \pm 37.51$ & $107.91 \pm 24.49$ & $116.42 \pm 39.05$ & 0.003 & $<0.001$ & 0.009 \\
\hline Mean pre-prandial glucose values (start of meal at 6:00 p.m.) (mg/dl) & $132.3 \pm 39.8$ & $142.5 \pm 49.2$ & $131.2 \pm 42.0$ & 0.888 & 1.000 & 1.000 \\
\hline Mean amount of hypoglycaemia rescue carbohydrates $(\mathrm{g})$ & $4.8 \pm 9.3$ & $39.2 \pm 22.5$ & $16.8 \pm 17.9$ & $<0.001$ & $<0.001$ & 0.001 \\
\hline Mean number of POC blood glucose measurements $\leq 70 \mathrm{mg} / \mathrm{dl}$ & $0.1 \pm 0.3$ & $1.2 \pm 1.0$ & $0.6 \pm 0.8$ & $<0.001$ & $<0.001$ & 0.002 \\
\hline
\end{tabular}




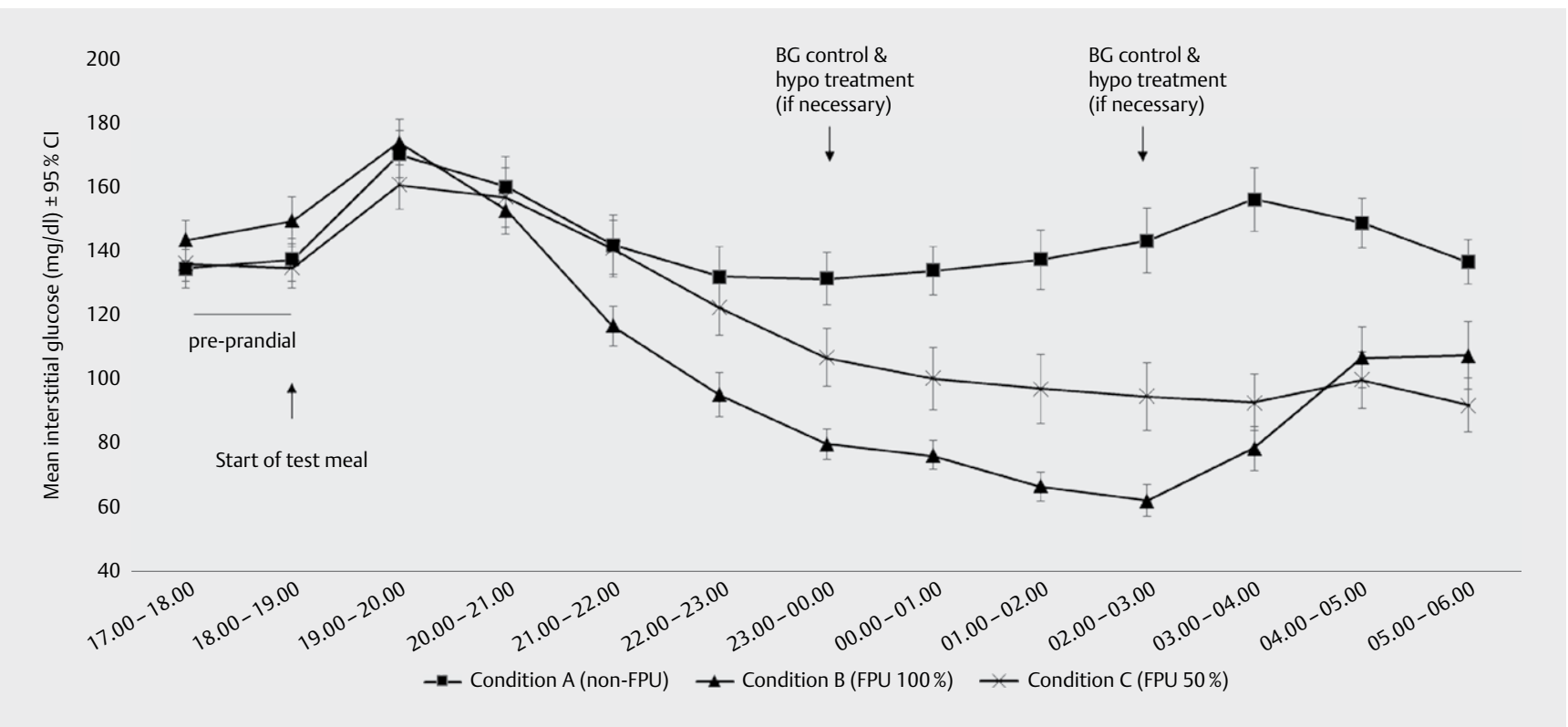

Fig. 3 Glucose course under the three test conditions between 5:00 p.m. (pre-prandial phase) and 6:00 a.m. the next day (confidence intervals that do not intersect mean a significant difference between the corresponding conditions).

by POC blood glucose readings. Particularly, nocturnal glucose had to be stabilized by approximately $40 \mathrm{~g}$ and $17 \mathrm{~g}$ of hypoglycemia rescue carbohydrates, respectively, using a high-dose algorithm and a low-dose algorithm. These results indicate that an adjustment of the prandial insulin dose for FPU in the tested dosage cannot be recommended for adults with type 1 diabetes. The tested dosage of prandial insulin was obviously too high to avoid postprandial hypoglycemia in the next 12 hours. The fact that the nadir of glucose was reached at $3.00 \mathrm{a} . \mathrm{m}$. and 4:00 am, respectively, in conditions $B$ and $C$, raises the question if the extension of the bolus over 8.00 hours is also disadvantageous besides a too high amount of additional bolus insulin due to the FPE adjustment. A shorter extension of the bolus may have avoided low glucose values between 3.00 am and 4:00 am in morning. The study had following strengths and limitations. The cross-over design of this study had the advantage of allowing all participants to serve as their own control group, as all participants took part in all three study conditions. The carry-over effect was also limited, as can be seen from the pre-prandial blood glucose values before the test meal. All participants were insulin pump users. Thus, it can be expected that the effect of delayed resorption of carbohydrates due to the relatively high-fat content could be controlled by delayed boluses. A further limitation of the study was that the carbohydrate-related amount of insulin was delayed over 4 hours in condition $A$ and over 8 hours in conditions $B$ and $C$.

A cross-over design also limits the number of meals that can be tested. The study tested only one meal, with a specific composition, in which $67.6 \%$ of the energy content were delivered by fats, $19.2 \%$ by proteins, and $13.2 \%$ by carbohydrates. In their review, Bell et al. [3] suggested that not only does the amount of fat and protein per se have a hyperglycemic effect, but also the composition of the macronutrients that can alter the demand of prandial insulin. Therefore, it is difficult to generalize findings to other fat- or protein-rich meals with varying compositions of these macronutrients.

In our study, the algorithms from Pańkowska et al. [11], which recommends the use of an insulin-to-FPU ratio equal to the CIR, and from Lee et al. [15], which recommends the use of an insulinto-FPU in a ratio equal to 0.5 of the CIR, lead to an over-insulinization with a high incidence of biochemical hypoglycemia. Outside an inpatient setting with close surveillance of nocturnal hypoglycemia, such algorithms can be qualified as dangerous.

Clearly, more research is needed to determine the amount of FPUs and insulin that should be taken into account when calculating pre-prandial insulin dosage.

A smart and promising research strategy for getting more evidence on FPU adjustment could be the use of closed-loop systems for such research questions on nutrition. Wolpert et al. [8] analyzed the dosing of prandial insulin after a low-fat and high-fat dinner while keeping the carbohydrate content equal in both study conditions in people with type 1 diabetes using a closed-loop system. They observed that on doubling the fat content of the dinner, prandial insulin doses increased from 9.0 insulin units to 12.6 insulin units $(+40 \%)$. This increase in prandial insulin dose in the high-fat condition in their closed-loop study was remarkably smaller than that observed in our study ( $+100 \%$ resp. $+200 \%)$. Wolpert et al. [8] also observed marked interindividual differences in the additional insulin requirements due to a high-fat content.

In summary, the results of this study could not demonstrate an advantage of post-prandial glucose excursion when using insulin for FPUs. In fact, using insulin for FPUs resulted in an over-insulinization with an elevated risk of hypoglycemia. Clearly, more research with sound methodology is needed before general adjustments of prandial insulin dosing for adults with type 1 diabetes can be established. 


\section{Authors Contributions}

All authors were involved in planning the study. EH and MK collected the data, TH and BLG monitored the correct conduct of the study. $\mathrm{NH}$ and $\mathrm{MK}$ analyzed the data. MK and $\mathrm{NH}$ wrote the manuscript. MK, $\mathrm{NH}$, and $\mathrm{TH}$ contributed to the discussion, reviewed and revised the manuscript. All authors have read and approved the final manuscript.

\section{Conflict of Interest}

All authors declare no competing interests.

\section{References}

[1] American Diabetes Association (ADA). 1. Improving care and promoting health in populations: Standards of medical care in diabetes - 2020. Diabetes Care 2020; 43: S7-13

[2] Haak T, Gölz S, Fritsche A et al. Therapy of Type 1 Diabetes. Abridged Version of the S3 Guideline (AWMF Register 2nd Edition). Exp Clin Endocrinol Diabetes 2019; 127: S27-38

[3] Bell KJ, Smart CE, Steil GM et al. Impact of fat, protein, and glycemic index on postprandial glucose control in type 1 diabetes: Implications for intensive diabetes management in the continuous glucose monitoring era. Diabetes Care 2015; 38: 1008-1015

[4] Carstensen S. Postprandiale blutglukoseverläufe in der (Insulinpumpen-) therapie des typ-1-diabetes. Einfluss der nahrungsbestandteile fett und eiweiß. Diabetologe 2012; 8: 213-221

[5] García-López JM, González-Rodriguez M, Pazos-Couselo M et al. Should the amounts of fat and protein be taken into consideration to calculate the lunch prandial insulin bolus? Results from a randomized crossover trial. Diabetes Technol Ther 2013; 15: 166-171

[6] Smart CE, Evans M, O'Connell SM et al. Both dietary protein and fat increase postprandial glucose excursions in children with type 1 diabetes, and the effect is additive. Diabetes Care 2013; 36: 3897-3902
[7] Pickup JC. Is insulin pump therapy effective in Type 1 diabetes? Diabetic Med 2019; 36: 269-278

[8] Wolpert HA, Atakov-Castillo A, Smith SA et al. Dietary fat acutely increases glucose concentrations and insulin requirements in patients with type 1 diabetes: implications for carbohydrate-based bolus dose calculation and intensive diabetes management. Diabetes Care 2013; 36: $810-816$

[9] Paterson M, Bell KJ, O'Connell SM et al. The role of dietary protein and fat in glycaemic control in type 1 diabetes: Implications for intensive diabetes management. Curr Diab Rep 2015; 15: 61

[10] Russell WR, Baka A, Björck I et al. Impact of diet composition on blood glucose regulation. Crit Rev Food Sci Nutr 2016; 56: 541-590

[11] Pańkowska E, Błazik M. Bolus calculator with nutrition database software, a new concept of prandial insulin programming for pump users. J Diabetes Sci Technol 2010; 4: 571-576

[12] Błazik M, Pańkowska E. The effect of bolus and food calculator Diabetics on glucose variability in children with type 1 diabetes treated with insulin pump: the results of RCT. Pediatr Diabetes 2012; 13 534-539

[13] Pankowska E, Blazik M, Groele L. Does the fat-protein meal increase postprandial glucose level in type 1 diabetes patients on insulin pump: the conclusion of a randomized study. Diabetes Technol Ther 2012; 14: $16-22$

[14] Kordonouri O, Hartmann R, Remus K et al. Benefit of supplementary fat plus protein counting as compared with conventional carbohydrate counting for insulin bolus calculation in children with pump therapy. Pediatr Diabetes 2012; 13: 540-544

[15] Lee SW, Cao M, Sajid S et al. The dual-wave bolus feature in continuous subcutaneous insulin infusion pumps controls prolonged post-prandial hyperglycaemia better than standard bolus in Type $1 \mathrm{dia}-$ betes. Diabetes Nutr Metab 2004; 17: 211-216

[16] Battelino T, Danne T, Bergenstal RM et al. Clinical targets for continuous glucose monitoring data interpretation: Recommendations from the international consensus on time in range. Diabetes Care 2019; 42: 1593-1603 